\title{
Enhanced production of periplasmic interferon alpha-2b by Escherichia coli using ion- exchange resin for in situ removal of acetate in the culture
}

\begin{abstract}
The possibility of using in situ addition of anion-exchange resin for the removal of acetate in the culture aimed at improving growth of E. coli and expression of periplasmic human interferon- $\alpha 2 b$ (PrIFN- $\alpha 2 b$ ) was studied in shake flask culture and stirred tank bioreactor. Different types of anion-exchange resin were evaluated and the concentration of anionexchange resin was optimized using response surface methodology. The addition of anionexchange resins reduced acetate accumulation in the culture, which in turn, improved growth of E. coli and enhanced PrIFN- $\alpha 2 b$ expression. The presence of anion-exchange resins did not influence the physiology of the cells. The weak base anion-exchange resins, which have higher affinity towards acetate, yielded higher PrIFN- $\alpha 2 b$ expression as compared to strong anion-exchange resins. High concentrations of anion-exchange resin showed inhibitory effect towards growth of E. coli as well as the expression of PrIFN- $\alpha 2 b$. The maximum yield of PrIFN- $\alpha 2 \mathrm{~b}$ in shake flask culture $(501.8 \mu \mathrm{g} / \mathrm{L})$ and stirred tank bioreactor $(578.8 \mu \mathrm{g} / \mathrm{L})$ was obtained at ion exchange resin (WA 30) concentration of $12.2 \mathrm{~g} / \mathrm{L}$. The production of PrIFN$\alpha 2 b$ in stirred tank bioreactor with the addition of ion exchange resin was about 1.8-fold higher than that obtained in fermentation without ion exchange resin $(318.4 \mu \mathrm{g} / \mathrm{L})$.
\end{abstract}

Keyword: Acetic acid; Adsorption; Anion-exchange resins; Bioreactors; Fermentation; Periplasmic interferon-alpha2b 